\title{
HEALTH LITERACY AMONG THAI ELDERLY POPULATION WITH TYPE-2 DIABETES LIVING IN RURALAREA OF THAILAND
}

\author{
Kantapong Prabsangob ${ }^{1}$, Ratana Somrongthong ${ }^{2}$, Ramesh Kumar ${ }^{3}$
}

${ }^{1}$ College of Allied Health Science, Suan Sunandha Rajabhat University, Thailand

${ }^{2}$ College of Public Health Sciences, Chulalongkorn University, Thailand

${ }^{3}$ Health Services Academy Islamabad Pakistan.

Correspondence: Professor Ratana Somrongthong, E-mail: sratana3@chula.ac.th

\begin{abstract}
Background: According to the World Health Organization, health literacy represents the cognitive and social skills, which determine the motivation and ability of individuals to gain access to, understand and use information in ways that promote and maintain good health with positive impact on health outcome.

Objective: This study aimed to describe the situation of health literacy among Thai elders with type-2 diabetes in a rural Thai community.

Methods: We conducted this mix-method study including quantitative and qualitative research approach was used through validated and reliable questionnaire adapted from Ishikawa. We did purposive sampling of 277 participants for quantitative, and conducted 12 in-depth interviews. Study was ethically approved from Chulalongkorn University Thailand.

Results: The average age of the participants was 68.62 years. The majority of them had income less than 5,001 Bath, and highest education level at elementary school. Most of them had been diagnosed with DM more than one year (ranged 1-56 years, average 10.05 years). The respondents had fair overall health literacy. Upon consideration as an aspect basis, the mean score of functional health literacy was 3.07 from 4.00 , the interactive health literacy was 2.28 , and the critical health literacy was 2.41. From the perspective of patients and caregivers, most patients received health-related information from public health officials. They do not like to talk to other diabetes patients about their diabetes. Most patients wanted to have someone who was willing to listen to their problem and help them solve it.
\end{abstract}

Conclusion: Study has concluded that the health literacy among Thai population suffering with diabetes was very poor and not found as per standards that need to be intervened.

Keywords: Health literacy, diabetes mellitus, elderly population

\section{Introduction}

Diabetes is a non-communicable disease with growing burden in developing countries (1). Nowadays, diabetes is causing many deaths around the world. According to World Health Organization (WHO), in year 2025, the world's population of people living with diabetes will be approximately 300 million and one in ten adults is suffering from diabetes across world $(2,3)$. In developed countries like; United States of America (USA), Australia and New Zealand, approximately 50 to 70 percent of people living with diabetes could control the blood glucose to an appropriate level. According to the Bureau of Policy and Strategy, the Ministry of Public Health in 2008 there was a continually increasing rate of diabetes patients reporting at hospitals during 1998 to 2008. A survey conducted by the National Health Security Office (NHSO) showed that only $35.6 \%$ of the diabetes patients met the glycemic goal of $\mathrm{HbA} 1 \mathrm{c}<7 \%$ in 2014
$(4,5)(6-8)$. Diabetic patients with lower health literacy tend to have less ability to control their blood glucose level as they do not understand health-related suggestions given by their doctors, resulting in less appropriate behavior in taking good care to their health (9-12).

Due to the increase numbers of the patients visiting doctors might effect on the quality of care provided by the physician. Elderly populations are usually neglected by their living places by their children and relatives. Hence, this study was imperative to discover the level of health literacy among elderly population with type-2 diabetes in rural Thailand.

\section{Methodology}

A mix-method study by adopting quantitative and qualitative method was used by interviewing 277 elderly population suffering from type-2 diabetes living in Bang Khonthi district of Samut Songkram province of 
Thailand. A validated, pretested and reliable questionnaire with 3-level Health Literacy Scale adopted from Ishikawa was used to assess health literacy level $(13,14)$. The patients respond to each question by rating from 1 , which means never to 4 , which means regularly. The higher score means that the patients have a higher health literacy, except the score result of basic health literacy in which the higher score means that the patients have a lower health literacy. Thus, scores on the health literacy ranges from 14 to 56 (15). Interpretations of the percentage score provided a measure of poor health literacy (25-60), fair health literacy (61-70), good health literacy (71-80), and very good health literacy (81 and above).

For a qualitative research method, observation and indepth interviews were used to collect data about perspectives of diabetes patients, caregivers, doctors, nurses, public health officers, and health literacy experts on health literacy promotion of sub-district health promotion hospitals in the Bang Khonthi district. Two persons from each group-diabetes patients, caregivers, doctors, nurses, public health officers, and health literacy experts-were selected for an in-depth interview. The total number of participants in the interview was 12 persons. The interview was tape-recorded. The researcher observed and recorded information obtained from the interview. In addition, an anthropological expert, who was an advisor making some recommendations, closely advised during an in-depth interview data collection process in order to receive appropriate and accurate information. Data was collected and analyzed by using content analysis to describe the promotion and perception of health literacy. Ethical approval was attained from the Chulalongkorn University Institutional Review Board on human rights prior to commencing the study.

\section{Results}

It was revealed that the majority of the participants were average 68.62 years old. The majority of them had income less than 5,001 (48.25\%) and highest education level at elementary school $(75.90 \%)$. Most of them had been diagnosed with DM for more than one year (ranged 1-56 years, average 10.05 years) as shown in Table 1. The respondents had fair overall health literacy $(67.06$ $\%$ : score 36.21 from 54 ). Upon consideration as an aspect basis, the mean score of functional health literacy was 3.07 , the interactive health literacy was 2.28 , and the critical health literacy was 2.41 , as shown in Table 1.
Table 1: Socio-demographic Characteristics and overall health literacy of respondents

\begin{tabular}{|c|l|l|l|}
\hline \multicolumn{1}{|c|}{ Characteristics } & \%/Average score & Mean & S.D. \\
\hline Socio-demographic & & & \\
\hline Age & & 68.62 y. & 6.73 \\
\hline Income less than 5,001 & $48.25 \%$ & & \\
\hline Element school level & $75.90 \%$ & & \\
\hline Duration of diabetes & & $10.05 \mathrm{y}$. & 8.10 \\
\hline $\begin{array}{c}\text { Health literacy } \\
\text { Functional health } \\
\text { literacy }\end{array}$ & 15.35 & 3.07 & 0.868 \\
\hline $\begin{array}{c}\text { Interactive health } \\
\text { literacy }\end{array}$ & 11.40 & 2.28 & 0.868 \\
\hline Critical health literacy & 9.64 & 2.41 & 0.968 \\
\hline Overall health literacy & $\mathbf{3 6 . 2 1 ~ ( 6 7 . 0 6 ~ \% ) ~}$ & $\mathbf{2 . 5 8}$ & $\mathbf{0 . 6 4 6}$ \\
\hline
\end{tabular}

All participants expressed satisfaction with participating in an in-depth interview. They wanted to express their opinion regarding the health literacy situation. The following is the result of observation and content analysis, categorized by groups of participants. Most patients receive health-related information from public health officials. The staffs will provide and distribute health knowledge documents to them. Every month, on the day the diabetes clinic is open, there will be staffs to lecture on health issues. For many patients, when they have a problem with insufficient understanding of health issues, they will consult health care providers. However, since most of them are elderly, they need help from relatives and friends who will take them to see a doctor and take their health information during the doctor visit. These friends and relatives will help take care of patients regarding food consumption and medication. Most patients do not have problems with receiving healthrelated information. However, the commonest problem is that, once they have received the health-related information, they do not care to read the document. In addition, regarding whatever they know from the information they often think and make decisions by themselves without any help to consider. They do not like to talk to other diabetes patients about their diabetes. Most patients want to have someone who is willing to listen to their problem and help them solve the problem.

"I know everyone is busy. I do not want to disturb them when I have a problem understanding health-related information because I will feel upset when they are not willing to help me. So, I try to do things on my own." (Patient N)

\section{Perspective of caregivers}

Most of the caregivers regularly receive health-related information and knowledge about diabetes care from health officials, physicians and nurses. Friends and relatives provide advice and assistance in the care of patients. However, many patients rarely follow their advice. They are ignorant towards the control of blood 
sugar and unhealthy eating habits. They like to do whatever they want without listening to others.

"My uncle is very pertinacious. I keep telling him do not do whatever the doctor prohibited, but he does not listen to me. He likes to do whatever he thinks is right." (Caregiver S)

\section{Perspective of doctors}

Although doctors do not have much time to talk to patients, whenever a patient has health problems, they are ready to help. However, most patients rarely ask the doctors questions regarding health problems. When the doctors ask them what the problem they have understanding health information is, most patients often said they do not have any problem. However, many patients usually like to do whatever is not -recommended by the doctor. They still consume unhealthy things such as sweet food. Doctors do not know that patients do not understand or do not care to follow their recommendations.

"I am concerned that many elderly patients may have a problem understanding the health information that I gave to them. I keep asking them if they have any problem or concern about what I just said to them. Most of them often said they are fine and do not have any problem. However, when they go home many of them do not follow my recommendations." (Doctor $\mathrm{Ch}$ )

\section{Perspective of nurses}

Nurses will provide health-related information and knowledge about diabetes care. They often advise and warn patients when they did something that might have a negative effect on their health. However, there are some patients who are not interested in the health-related information. They do not care about their health problems. They also do not care to take care of their own health.

"I know some patients do not like me because I keep telling them to do this or not to do that. Since they are elderly, they might not like a young person like me giving them orders. They may feel frustrated when I blame them for the wrong things they did." (Nurse P)

\section{Perspective of public health officials}

Every month public health officials will visit diabetes patients at home in the area for which they are responsible, especially those who have problems taking care of themselves and need health providers to help them at home. Health-related information and knowledge about diabetes care are provided to patients and caregivers when they come to receive services at the hospital. The public health officials make a lot of effort to find out if there is anything that makes it difficult for the patients to perceive and understand the health information. From their experience, they found that many patients have a good understanding of diabetes, especially those who have had diabetes for a long time. However, even when they know what is bad for their health, they still do it.

"Many elderly patients know a lot about diabetes care because they have received health-related information and knowledge about diabetes care for many years. However, some patients still do whatever they know may have serious effects on their health such as eating a lot of sweet food." (Public health official R)

\section{Perspective of health literacy experts}

At hospitals, health providers often focus on educating diabetes patients. They think that diabetes patients with good knowledge will perform well in maintaining good health. In fact, in the definition of health, patients have to be motivated to perceive and understand health information and be encouraged to use this information to their advantage. Even if the patients are knowledgeable, as long as there is no motivation, they will not practice good self-care behavior.

"For many patients who have good knowledge about diabetes care, they still do not practice good self-care behavior because they have no motivation to do it. Therefore, for promoting patients' health literacy, we have to know how to motivate them to access, understand, and use health-related information to improve their health." (Expert B)

\section{Discussion}

The result that elders with type-2 diabetes in Bang Khonthi District, Samut Songkram Province, Thailand, had fair health literacy may be because of their old age, low education and low income. This was in accordance with Schillinger and Nutbeam which had proven that limited health literacy is common among low-income and poorly educated elderly patients with diabetes $(8,11)$. When considering each aspect of health literacy, we found that interactive health literacy had the lowest mean score compared with functional and critical health literacy scores. According to the definition of health literacy, interactive health literacy refers to being literate in basic health, cognitive and social skills used in participating in social activities and selecting updated information for improving health behavior. When interactive health literacy is low, it means diabetes patients have less communication with others regarding their health and diabetes. This was in accordance with the in-depth interview which showed that the patients did not like to talk with others about their diabetes. This is similar to the findings of previous studies which revealed that diabetes patients with lower health literacy tend to have less ability to understand health-related suggestions given by their doctors. As a result, they seldom communicate with doctors $(9,10,11,12,16)$.

According to the in-depth interview, elders with type-2 diabetes want to communicate with others who are willing to listen to their problem and help them. However, caregivers and health care providers said that they make a lot of effort to take care of these diabetes patients. This means the way caregivers and health care providers did to the patients is not the thing that the patients want. Both of them have different viewpoints towards diabetes care. In addition, the study revealed that health literacy was positively associated with patient experience of primary care attributes, especially regarding longitudinality and 
comprehensiveness, among Japanese people who had a usual source of care. Furthermore, among the levels of health literacy, communicative and critical health literacy were associated with patient experience of primary care (17).

The study revealed that the social and cultural values of Thai people had an influence on their viewpoints towards diabetes. The word "diabetes" in the understanding of Thai people is the disease that is explained by a high level of blood sugar in the body until there is sugar in the urine. Thus, Thai patients try not to eat much carbohydrate and sweets, hoping to reduce their blood sugar level. Many are of the view that diabetes is as a result of their sin from a past life or this life, and thus they could not escape from it. This belief has influenced these patients to manage their health quite well. However, control of diets is a harder action for many patients. This is especially so in Thailand which is very prosperous in fruits, even with the belief in Buddhism which teaches moderation and sufficiency. The idiom, "eating to live, not living to eat", could explain the way of Thai people in releasing themselves from the sins of the past and present lives by reducing or removing meats in their meals and following vegetarian practices. These practices are good for the health condition of diabetes patients. Praying, practicing meditation, and following Buddhist ways can help diabetes patients remain calmer, less stressed and have a good reminder of what to do to take care of their health. These all help to bring about smoother and more effective management of diabetes treatment (18).

Conversely, Thai society respects seniority. Younger persons should respect older persons. In hospitals, there are young doctors and nurses who take care of diabetes patients who are much older. The way doctors and nurses tell the patients to follow their instructions strictly may cause dissatisfaction and adverse reaction from these old patients who may feel disrespected. The study found that during treatment of diabetes, if doctors behave in a way that makes old patients feel inferior, for example, ordering the patients to follow their instructions without allowing them to express any feelings or opinions, the doctors may not be able to receive sufficiently useful information from the patients, may not receive good participation from the patients, or the patients may decide to receive a treatment with a new doctor or at other hospitals (15). Some patients feel that they do not receive good respect from medical staff; some feel irritated and annoyed; some feel that they are not well cared for by medical staff. These influence them to seek out new doctors, new hospitals or other alternative treatments such as herbal treatment or traditional treatment. This can cause the diabetic condition to get worse.

\section{Conclusion}

The health literacy among Thai population was not up to the standards that need to be intervened to promote better communication between them and doctors and their behavior.

Acknowledgements: The author would like to thank Chulalongkorn University, Bangkok, Thailand for financial support.

\section{References}

1. Gastaldelli. A. Role of beta-cell dysfunction, ectopic fat accumulation and insulin resistance in the pathogenesis of type 2 diabetes mellitus. Diabetes Res Clin Pract.2011; 93(1): S60-65.

2. World Health Organization. Country and regional data on diabetes. Available at http:// http://www.who.int/diabetes/facts/world_figures len/.

3. World Health Organization. World Health Statistics 2012: World Health Organization. A $\vee$ a i I a b I e a $\mathrm{t}$ http://www.who.int/gho/publications/world_heal th_statistics/2012/en/.

4. Aekplakorn. W, Chariyalertsak. S, Kessomboon. $P$, Sangthong. R, Inthawong. R, Putwatana.P, et al. Prevalence and management of diabetes and metabolic risk factors in Thai adults: The Thai National Health Examination Survey IV, 2009. Diabetes Care. 2011; 34(9):1980-5.

5. Reutrakul. S, Deerochanawong. C. Diabetes in Thailand: Status and Policy. Curr Diab Rep.2016; 16(3):28.

6. Nutbeam. D. Health literacy as a public health goal: A challenge for contemporary health education and communication strategies into the 21st century. Health promotion international. 2000; 15(3):259-267.

7. Nutbeam. D. Health Literacy and adolescents: a framework and agenda for future research. Health Education Research. Published by Oxford University Press. 2008; 23(5).

8. Nutbeam. D. The evolving concept of health literacy. Social Science \& Medicine. 2008; 67:2072-2078.

9. Ad Hoc Committee on Health Literacy for the Council on Scientific Affairs, American Medical Association. Health Literacy. Report of the Council on Scientific Affairs. JAMA. 1999; 281(6):552-557.

10. Gazmararian. J.A, Baker. D.W, Williams. M.V, Parker. R.M, Scott. T.L, Green. D.C, Fehrenbach. S.N, Ren. J, Koplan. J.P. Health literacy among Medicare enrollees in managed care organization. JAMA. 1999; 281:545-551.

11. Schillinger. D, Grumbach. K, Piette. J, Wang. F, Osmond. D, Daher. C, Palacios. J, Sullivan. D.G, Bindman. B.A. Association of Health Literacy with Diabetes Outcomes. American Medical Association. 2002; 288: 475-482.

12. Williams. M.V, Baker. D.W, Parker. R.M, Nurss JR. Relationship of functional health literacy to patients' knowledge of their chromic disease: A study of patients with hypertension and 
diabetes. Arch Intern Med. 1998; 158:166-172.

13. Ishikawa. H, Takeuchi. T, Yano. E. Measuring functional, communicative, and critical health literacy among diabetic patients. Diabetes Care 2008; 31:874-879.

14. Suka. M, Odajima. T, Kasai. M, Igarashi. A, Ishikawa. H, Kusama. $M$ et al. The 14-item health literacy scale for Japanese adults (HLS14). Environ Health Prev Med. 2013; 18(5):407415.

15. Chananya. K, Wantana. M, Poolsuk. J. Relationships of Health Literacy and Knowledge about Antiretroviral Therapy to Medication Adherence among People Living with HIV. Kuakarun Journal of Nursing. 2014; 21(2):211227.

16. Kantapong P. (2016). Relationships of Health Literacy Diabetes Knowledge and Social Support to Self-Care Behavior among Type 2 Diabetic Patients. International Journal of Health and Medical Sciences. 2016; 2(3): 68-72.

17. Aoki. T, Inoue. M. Association between health literacy and patient experience of primary care attributes: A cross-sectional study in Japan. PLoS ONE. 2017; 12(9): e0184565. https://doi.org/10.1371/journal.pone.0184565

18. Sowattanangoon. N, Kotchabhakdi. N, Petrie J.K. The influence of Thai culture on diabetes perceptions and management. Diabetes Research and Clinical Practice. 2009; 84:245251. 